\title{
Re-expansion pulmonary edema following a pneumothorax drainage in a patient with COVID-19
}

\author{
Kosaku Komiya*, Ryosuke Hamanaka, Hisayuki Shuto, Hiroki Yoshikawa, Atsushi Yokoyama, \\ Kazufumi Hiramatsu and Jun-ichi Kadota
}

\begin{abstract}
Background: Re-expansion pulmonary edema is an uncommon complication following drainage of a pneumothorax or pleural effusion. While pneumothorax is noted to complicate COVID-19 patients, no case of COVID-19 developing re-expansion pulmonary edema has been reported.

Case representation: A man in his early $40 \mathrm{~s}$ without a smoking history and underlying pulmonary diseases suddenly complained of left chest pain with dyspnea 1 day after being diagnosed with COVID-19. Chest X-ray revealed pneumothorax in the left lung field, and a chest tube was inserted into the intrathoracic space without negative pressure $9 \mathrm{~h}$ after the onset of chest pain, resulting in the disappearance of respiratory symptoms; however, $2 \mathrm{~h}$ thereafter, dyspnea recurred with lower oxygenation status. Chest $\mathrm{X}$-ray revealed improvement of collapse but extensive infiltration in the expanded lung. Therefore, the patient was diagnosed with re-expansion pulmonary edema, and his dyspnea and oxygenation status gradually improved without any intervention, such as steroid administration. Abnormal lung images also gradually improved within several days.
\end{abstract}

Conclusions: This case highlights the rare presentation of re-expansion pulmonary edema following pneumothorax drainage in a patient with COVID-19, which recovered without requiring treatment for viral pneumonia. Differentiating re-expansion pulmonary edema from viral pneumonia is crucial to prevent unnecessary medication for COVID-19 pneumonia and pneumothorax.

Keywords: Re-expansion pulmonary edema, Pneumothorax, COVID-19

\section{Background}

Re-expansion pulmonary edema following pneumothorax or pleural effusion drainage has already been reported for decades, with an incidence of approximately $1 \%$ [1]. Although its pathophysiology remains poorly understood, re-expansion pulmonary edema is believed to be caused by increased permeability of the pulmonary capillaries through inflammatory reactions. Re-expansion

*Correspondence: komiyakh1@oita-u.ac.jp

Respiratory Medicine and Infectious Diseases, Faculty of Medicine, Oita

University, 1-1 Idaigaoka, Hasama-machi, Yufu, Oita 879-5593, Japan and reperfusion of a previously collapsed lung tissue may cause inflammations with reactive oxygen species and superoxide radicals and thereby may increase the capillary permeability $[2,3]$. For other mechanisms, increased pulmonary hydrostatic pressure caused by venous return, pressure-induced mechanical disruption of the alveolar capillaries and decreased functional surfactant levels may also be suggested to result in re-expansion pulmonary edema [4].

Pneumothorax has been reported as a complication in patients with COVID-19, requiring hospital admission, and may be associated with increased mortality [5, 
6]. A recent large observational study conducted in the United Kingdom revealed that 1283 (0.97\%) of 131,679 patients aged $>18$ years, who were admitted to hospitals for COVID-19, had pneumothorax at some stages during their admission [7]. The incidence differed between groups according to the level of respiratory support: $0.16 \%$ of patients not requiring supplemental oxygen, $0.56 \%$ of patients requiring oxygen without pressure support, $0.96 \%$ of patients treated with non-invasive respiratory support and $6.1 \%$ of patients who received invasive ventilation for pneumothorax. Multivariate analysis in this study showed that male sex, smoking, chronic pulmonary disease and invasive ventilation were associated with increased risk of pneumothorax.

Considering that approximately 2 billion people have already been infected with severe acute respiratory syndrome coronavirus 2 (SARS-CoV-2) worldwide, a certain number of patients with COVID-19 and pneumothorax should have suffered from re-expansion pulmonary edema following a drainage procedure. However, no such case has been reported. Herein, we present the case of a patient with COVID-19 diagnosed with re-expansion pulmonary edema following pneumothorax drainage and discuss the importance of differentiating re-expansion pulmonary edema from COVID-19 pneumonia.

\section{Case presentation}

A man in his early 40s had a low-grade fever and was positive for SARS-CoV-2 in the polymerase chain reaction result 5 days after. He did not have a smoking history and underlying pulmonary diseases. Due to no symptoms except for the low-grade fever, chest X-ray had not been performed at diagnosis of COVID-19. However, 1 day after diagnosis, he suddenly complained of left chest pain with dyspnea and visited our hospital. Physical examination revealed a body temperature of $37.7^{\circ} \mathrm{C}$, an oxygen saturation $\left(\mathrm{SpO}_{2}\right)$ of $93 \%$ without supplemental oxygenation, blood pressure of $117 / 81 \mathrm{mmHg}$ and heart rate of 99 beats/min. Laboratory tests revealed a normal leukocyte count (7320 cells/ $\mu \mathrm{L})$ and slightly elevated serum $C$-reactive protein levels $(0.30 \mathrm{mg} / \mathrm{dL})$. Chest X-ray revealed significant pneumothorax in the left lung field (Fig. 1A), with $78 \%$ of the Light index [8]. Chest computed tomography $(\mathrm{CT})$ performed to determine appropriate thoracic space for drainage tube revealed left lung collapse without pneumomediastinum and patchy ground-glass opacity (GGO) in the right lower lobe. We inserted a chest drainage tube (20 Fr) into the intrathoracic space without negative pressure $9 \mathrm{~h}$ after he reported chest pain and his respiratory symptoms disappeared and $\mathrm{SpO}_{2}$ improved to 98\%; however, after $2 \mathrm{~h}$, he re-experienced dyspnea with a lower $\mathrm{SpO}_{2}$ of $92 \%$. Chest X-ray revealed an improved collapse but infiltration of the expanded lung. Chest CT performed on the following day to distinguish re-expansion pulmonary edema from COVID-19 pneumonia revealed extensive consolidation with ground-glass opacities in all lobes of the expanded left lung (Fig. 1B). No significant morphological abnormality as a risk for pneumothorax has been observed. Re-expansion pulmonary edema was diagnosed instead of COVID-19 pneumonia progression based on this clinical course. His dyspnea and oxygenation status gradually improved without any intervention, including supplemental oxygen, diuretics or steroid administration, and abnormal lung involvements observed in the chest $\mathrm{CT}$ were diminished 7 days after the chest drainage procedure (Fig. 1C).

\section{Discussion and conclusions}

Herein, we present a case of re-expansion pulmonary edema following pneumothorax drainage with COVID19. No significant lung abnormality including bullae has been observed for the risk for pneumothorax in chest CT. SARS-CoV-2 infection might have been a trigger for pneumothorax, but no clear evidence was obtained in this case, although pneumonia was not extensive.

First, whether abnormal involvements in the left lung represent re-expansion pulmonary edema or COVID19 pneumonia needs further investigation. Symptoms of re-expansion pulmonary edema include chest discomfort, persistent severe cough, production of frothy sputum and dyspnea, which are commonly observed in patients with COVID-19. However, these symptoms have been reported to start within $1-2 \mathrm{~h}$ in $64 \%$ of patients with re-expansion pulmonary edema [4]. Dyspnea with decreased oxygenation status occurred $2 \mathrm{~h}$ after a drainage procedure, which is clinically compatible with re-expansion pulmonary edema. Furthermore, radiological features in the left lung after expansion do not appear to be COVID-19 pneumonia. Typical CT findings of COVID-19 pneumonia are predominantly peripheral patchy GGOs and/or consolidations, and vascular enlargement is commonly reported [9]. In this patient, consolidation was predominantly observed in the central field of the left upper lobe without vascular enlargement, which may support hydrostatic edema [10], whereas a patchy GGO in the right lower lobe is consistent with COVID-19 pneumonia. Moreover, the extensive consolidation with GGO has almost disappeared within several days, which is in agreement with the characteristics of reexpansion pulmonary edema. As the majority of patients with re-expansion pulmonary edema completely recover within 5-7 days [3], the extent of lung abnormalities caused by COVID-19 peaks at days 6-11 and gradually disappears [11]. The course of rapid improvement also supports that abnormal images were caused by re-expansion pulmonary edema. 


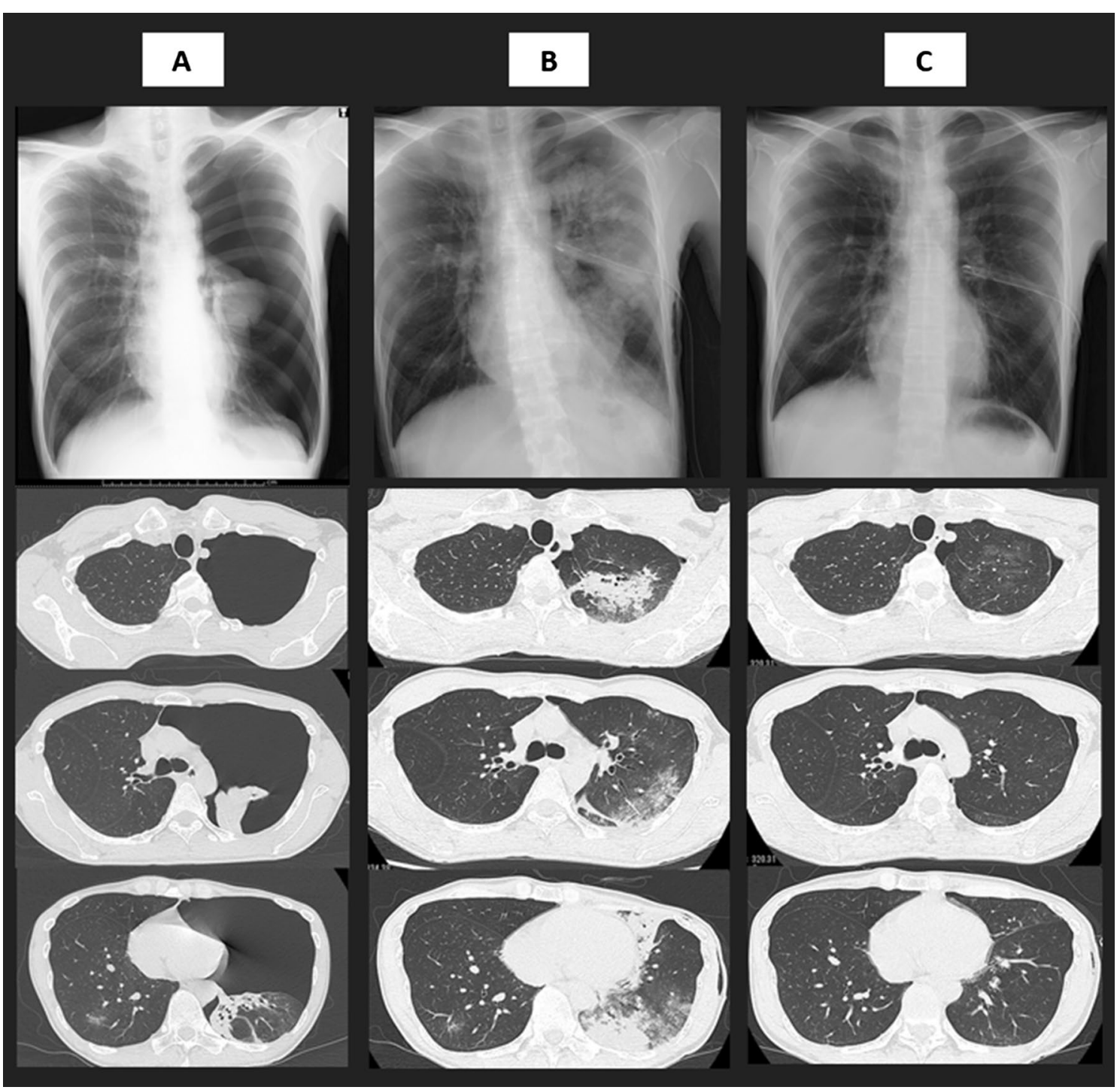

Fig. 1 Chest X-ray and thin-section computed tomography at upper lobes, carina and lower lobes levels on admission (A), 1 day after thoracic drainage procedure (B) and 7 days after thoracic drainage procedure (C)

Re-expansion pulmonary edema following a pneumothorax or pleural effusion drainage rarely occurs [1]. Proposed risk factors include collapse duration of $>72 \mathrm{~h}$ and the application of high negative pressures during thoracic drainage $\left(>20 \mathrm{~cm} \mathrm{H}_{2} \mathrm{O}\right)$ [4]. Although these risk factors were not observed in the present case, he suffered from re-expansion pulmonary edema. In a small retrospective study [12], pneumothorax size was associated with the occurrence of re-expansion pulmonary edema. The large size of pneumothorax in the current case might have accelerated the development of re-expansion pulmonary edema. The SARS-CoV-2 infection could be associated with an increased risk for re-expansion pulmonary edema. SARS-CoV-2 infection is known to cause cytokine-mediated endothelialitis in the pulmonary vascular system and increases transepithelial permeability [13]. Hypoxia also decreases active sodium transport across the alveolar wall and reduces alveolar fluid reabsorption [14]. In an acid aspiration lung injury mouse model, knockout of angiotensinconverting enzyme 2 (ACE2), a known main receptor of SARS-CoV-2, has been reported to worsen pulmonary edema [15]. Indeed, COVID-19 infection causes a functional ACE2 deficiency in the lung and increases the production of proinflammatory cytokines and extends half-time of some cytokines including bradykinin [16]. 
Moreover, COVID-19 is characterized by an increased incidence of pulmonary embolism [17]. This phenomenon increases the capillary hydrostatic pressure and pulmonary vascular resistance, as well as precapillary and postcapillary resistance [18], which may result in the development of pulmonary edema. However, no evidence has been established on whether the risk of reexpansion pulmonary edema is related to the severity of COVID-19 pneumonia. At least, physicians need to be aware of general risk factors for re-expansion pulmonary edema including long duration of collapse, pneumothorax size and high negative pressure for drainage even in a patient with COVID-19 and pneumothorax.

Whether re-expansion pulmonary edema following thoracic drainage is more prone to occur in patients with COVID-19 than that in the general population, the incidence would be underestimated because no case of re-expansion pulmonary edema with COVID19 has been reported. Since pneumothorax is more likely to occur in patients with severe COVID-19 pneumonia requiring invasive ventilation, distinguishing re-expansion pulmonary edema from extensive COVID-19 pneumonia would be challenging. However, if re-expansion pulmonary edema is misdiagnosed as progressive COVID-19 pneumonia and then escalated medication is administered including additional corticosteroid, it would worsen the prognosis of pneumothorax. Differentiating possibly underestimated re-expansion pulmonary edema following thoracic drainage from COVID-19 pneumonia could effectively improve the disease prognosis.

\section{Abbreviations}

ACE2: Angiotensin-converting enzyme 2; COVID-19: Coronavirus disease 2019; CT: Computed tomography; GGO: Ground-glass opacity; SARS-CoV-2: Severe acute respiratory syndrome coronavirus 2 .

\section{Acknowledgements}

The authors thank Drs. Ryosuke Masui, Marimu Yamanaka, Ai Tanaka, Izumi Yamatani, Hiroyuki Matsumoto and Mari Yamasue for clinical advice.

\section{Authors' contributions}

$\mathrm{RH}, \mathrm{HS}, \mathrm{HY}$ and $\mathrm{AY}$ contributed to the data collection. $\mathrm{RH}, \mathrm{HS}, \mathrm{HY}, \mathrm{AY}, \mathrm{KK}$, $\mathrm{KH}$, and JK drafted the manuscript. All authors have read and approved the manuscript.

\section{Funding}

This research did not receive any specific grant from funding agencies in the public, commercial, or not-for-profit sectors,

\section{Availability of data and materials}

Data are available in this manuscript.

\section{Declarations}

Ethics approval and consent to participate

Not applicable due to a case report not including personally identifiable information.
Consent for publication

Written informed consent to publish this report was obtained from the patient.

\section{Competing interests}

All authors have stated explicitly that there are no conflict of interest in connection with this article.

Received: 2 August 2021 Accepted: 6 September 2021

Published online: 16 September 2021

\section{References}

1. Echevarria C, Twomey D, Dunning J, Chanda B. Does re-expansion pulmonary oedema exist? Interact Cardiovasc Thorac Surg. 2008;7(3):485-9.

2. Kasmani R, Irani F, Okoli K, Mahajan V. Re-expansion pulmonary edema following thoracentesis. CMAJ Can Med Assoc J. 2010;182(18):2000-2.

3. Sherman SC. Reexpansion pulmonary edema: a case report and review of the current literature. J Emerg Med. 2003;24(1):23-7.

4. Mahfood S, Hix WR, Aaron BL, Blaes P, Watson DC. Reexpansion pulmonary edema. Ann Thorac Surg. 1988;45(3):340-5.

5. Miró Ò, Llorens P, Jiménez S, Piñera P, Burillo-Putze G, Martín A, MartínSánchez FJ, García-Lamberetchs EJ, Jacob J, Alquézar-Arbé A, et al. Frequency, risk factors, clinical characteristics, and outcomes of spontaneous pneumothorax in patients with coronavirus disease 2019: a case-control. Emerg Med Based Multicenter Study Chest. 2021;159(3):1241-55.

6. Wang XH, Duan J, Han X, Liu X, Zhou J, Wang X, Zhu L, Mou H, Guo S. High incidence and mortality of pneumothorax in critically III patients with COVID-19. Heart Lung. 2021;50(1):37-43.

7. Marciniak SJ, Farrell J, Rostron A, Smith I, Openshaw PJM, Baillie JK, Docherty A, Semple MG. COVID-19 Pneumothorax in the United Kingdom: a prospective observational study using the ISARIC WHO clinical characterisation protocol. Eur Respir J 2021

8. Light RW. Management of spontaneous pneumothorax. Am Rev Respir Dis. 1993;148(1):245-8.

9. Ojha V, Mani A, Pandey NN, Sharma S, Kumar S. CT in coronavirus disease 2019 (COVID-19): a systematic review of chest CT findings in 4410 adult patients. Eur Radiol. 2020;30:1-10.

10. Storto ML, Kee ST, Golden JA, Webb WR. Hydrostatic pulmonary edema: high-resolution CT findings. AJR Am J Roentgenol. 1995;165(4):817-20.

11. Wang Y, Dong C, Hu Y, Li C, Ren Q, Zhang X, Shi H, Zhou M. Temporal changes of $C T$ findings in 90 patients with COVID-19 pneumonia: a longitudinal study. Radiology. 2020;296(2):E55-e64.

12. Haga T, Kurihara M, Kataoka H. Risk for re-expansion pulmonary edema following spontaneous pneumothorax. Surg Today. 2014;44(10):1823-7.

13. Ackermann M, Verleden SE, Kuehnel M, Haverich A, Welte T, Laenger F, Vanstapel A, Werlein C, Stark H, Tzankov A, et al. Pulmonary vascular endothelialitis, thrombosis, and angiogenesis in Covid-19. N Engl J Med. 2020;383(2):120-8.

14. Vivona ML, Matthay M, Chabaud MB, Friedlander G, Clerici C. Hypoxia reduces alveolar epithelial sodium and fluid transport in rats: reversal by beta-adrenergic agonist treatment. Am J Respir Cell Mol Biol. 2001;25(5):554-61.

15. Imai Y, Kuba K, Rao S, Huan Y, Guo F, Guan B, Yang P, Sarao R, Wada T, Leong$\mathrm{Poi} \mathrm{H}$, et al. Angiotensin-converting enzyme 2 protects from severe acute lung failure. Nature. 2005;436(7047):112-6.

16. de Maat S, de Mast Q, Danser AHJ, van de Veerdonk FL, Maas C. Impaired breakdown of bradykinin and its metabolites as a possible cause for pulmonary edema in COVID-19 infection. Semin Thromb Hemost. 2020;46(7):835-7.

17. Bikdeli B, Madhavan MV, Jimenez D, Chuich T, Dreyfus I, Driggin E, Nigoghossian C, Ageno W, Madjid M, Guo Y, et al. COVID-19 and thrombotic or thromboembolic disease: implications for prevention, antithrombotic therapy, and follow-up: JACC state-of-the-art review. J Am Coll Cardiol. 2020;75(23):2950-73.

18. Evlakhov VI, Poyassov IZ, Ovsyannikov VI. Pulmonary microcirculation in experimental model of pulmonary thromboembolism under conditions of a-adrenoceptor blockade. Bull Exp Biol Med. 2019;166(3):313-6.

\section{Publisher's Note}

Springer Nature remains neutral with regard to jurisdictional claims in published maps and institutional affiliations. 